\title{
NUEVOS HITOS EN LA ARQUEOLOGÍA DE CANAÁN VI CAMPAÑA DE EXCAVACIONES EN TEL HATSOR
}

M. ${ }^{a}$ TERESA RUBIATO DÍAZ Universidad Complutense. Madrid

Es ya habitual comenzar la crónica de alcance de las excavaciones en Tel Hatsor haciendo referencia a la riqueza de los hallazgos y al interés de los datos que este yacimiento siempre ha proporcionado para el esclarecimiento de los múltiples enigmas históricos que aún ofrecen los períodos cananeos e israelitas. Pero lo cierto es que la VI campaña de excavaciones en Tel Hatsor (junio-agosto 1995) ha sido la más fructífera de las hasta ahora realizadas, tanto por lo que se refiere al abundante, variado y excelente caudal de datos como al ingente número de hallazgos, algunos de ellos únicos hasta ahora en la arqueología de Canaán.

La actividad se centró, según el programa de trabajo, en dos áreas principales: el área $\mathrm{A}$, en el centro neurálgico de la acrópolis, y el área $M$, en la ladera norte de la propia ciudad alta.

En el área A -la mayor en extensión de las dos áreas de excavación- aún continuaron los trabajos de excavación de restos del período israelita. Ha de tenerse en cuenta que los ocupantes de la ciudad post-cananea se instalaron en y sobre los restos del último trazado de la Hatsor del Bronce, cavando en ellos con frecuencia los cimientos de sus construcciones. Por ello con la misma frecuencia el excavador se encuentra trabajando simultáneamente en estratos de distintas épocas, de los que no siempre el más profundo es el más antiguo. Varios silos del período israelita, por ejemplo, aparecen excavados en dependencias del gran palacio cananeo por los posteriores habitantes de Hatsor, siglos después de la gran destrucción e incendio. Lo mismo ocurre en el caso de almacenes, talleres y casas.

Los cimientos de un gran edificio público del período israelita se encontraron en la zona occidental del área A. Ya en campañas anteriores los restos del palacio cananeo, que aparecían inmediatamente bajo la superficie, se veían perturbados bruscamente por un 
muro de piedras que corría en ligera pero marcada diagonal con respecto a los muros de adobe del palacio. Solamente el muro meridional del edificio israelita, de treinta metros de largo, fue descubierto en su totalidad. Del muro oriental se excavó un segmento de más de veinte metros. Esta estructura - gran parte de la cual está ocupada por el actual aparcamiento de coches y autocarestiene una planta prácticamente cuadrada. Los cimientos tienen unos tres metros de espesor y unos dos metros de profundidad. Esos cimientos están insertos directamente en los restos de adobe del palacio cananeo. Según se ha podido ir deduciendo del análisis de los resultados de campañas anteriores, los restos del palacio cananeo de Hatsor aparecen directamente bajo la superficie en muchos puntos porque el área que ocupaba fue durante siglos una especie de plaza abierta y despejada; en otros puntos del centro de la acrópolis otras dependencias del palacio recibieron, como queda dicho, la intrusión de edificaciones ya desde el siglo XII y en especial a partir del siglo $\mathrm{X}$ a. C. Afortunadamente, el gran edificio israelita excavado en la presente campaña no alcanza con sus cimientos ni los suelos ni otros puntos neurálgicos del palacio cananeo. Este edificio israelita, del que no ha aparecido ningún suelo, puede no obstante ser datado con seguridad en las postrimerías del siglo VIII a. C., el estrato V de Y. Yadin. Esta fecha se infiere de la cerámica encontrada en un único pequeño fragmento de suelo, de la cerámica recogida en las zanjas de los cimientos y principalmente por la cerámica del siglo VIII encontrada en los suelos de un edificio menor adyacente, que fueron cortados por la gran estructura que nos ocupa.

Este gran edificio es la mayor construcción israelita encontrada hasta la fecha en Hatsor. Por comparación, las dimensiones de la ciudadela del área B, excavada por la expedición de Y. Yadin en los años cincuenta (estratos VIII-V) tiene unos $22 \times 25$ metros, sus muros son de dos metros de ancho, y la profundidad de sus cimientos aproximadamente de un metro. El edificio israelita excavado por nosotros este año arroja nueva luz sobre la Hatsor del siglo VIII a. C.: es un índice más de que incluso en esos turbulentos años, que hasta ahora habían sido descritos como un período de declive en comparación con los tiempos dorados del siglo IX a. C., Hatsor mantenía su fuerza y su riqueza. Los desnudos cimientos del gran edificio público a que nos estamos refiriendo nos sugieren, dicho sea a título de impresión muy personal, que la ambiciosa estructura no 
sólo no fue jamás utilizada, sino que los acontecimientos del momento - quizás la inminencia del ataque asirio- hicieron que las fuerzas de Hatsor se concentraran en el reparación de la ciudadela del área $\mathrm{B}$ y otros puntos defensivos de los bordes de la acrópolis, abandonando la propia construcción del edificio del área A. No es explicable de otra forma no ya la ausencia de suelos y de todo vestigio de ocupación, sino la uniforme altura de los cimientos. El proyecto debió de ser, como queda dicho, muy ambicioso y arquitectónicamente avanzado: a diferencia de otros edificios del período israelita de Hatsor, en éste sus arquitectos parecen haber incorporado una nueva técnica de construcción antisísmica. Esta zona de la Alta Galilea es, en efecto, una región telúrica de frecuentes temblores de tierra en ocasiones catastróficos, como el que destruyó casi totalmente el estrato VI de la acrópolis en el siglo VIII a. C. Para la cimentación del edificio emplearon grandes piedras en la hilera más profunda, estabilizadas por otras insertadas entre y sobre ellas de forma que creaban una resistente plataforma, pero no tan rígida que no soportara posibles temblores sísmicos; sobre esa firme base se elevaban otras hileras de piedras hasta la citada altura de dos metros.

Otros vestigios de la Hatsor israelita han sido investigados en la presente campaña, en la zona adyacente al gran edificio de tres naves del siglo IX a. C. (contemporáneo del edificio de pilares) excavado en las tres campañas anteriores. Este siglo IX a. C. representa el cenit de la prosperidad de la Hatsor de la monarquía israelita: la superficie de la ciudad se dobló con respecto a la del siglo $\mathrm{X}$ a. C., se ampliaron y consolidaron sus fortificaciones, se excavó el gran sistema hidráulico y se erigieron varias grandes estructuras públicas. Los vestigios excavados en 1995 pertenecen sobre todo a una hermosa casa ocupada durante largo tiempo que ha proporcionado abundantes hallazgos.

En el período de la monarquía unida, siglo $\mathrm{x}$ a. C., la ciudad era más pequeña que la del período subsiguiente, sin embargo, esta campaña ha proporcionado datos muy relevantes para el estudio de ese período y para la determinación de la completa secuencia de ocupación de la Hatsor israelita.

Los restos de los siglos XI y XII a. C. (período del asentamiento israelita) que se encuentran en Hatsor son de naturaleza más pobre y consisten principalmente en muros fragmentarios, hoyos llenos de 
ceniza, restos de piedras de mortero y un limitado conjunto de cerámica. Estos pobres restos son, sin embargo, de un altísimo interés, tanto en Hatsor como en todos los demás yacimientos arqueológicos de la zona. La identidad de los ocupantes de Hatsor durante este período (¿se trata de los israelitas?) y su relación con los que destruyeron la Hatsor cananea (ison los responsables de esa destrucción?) son cuestiones que permanecen abiertas.

Los restos de la Hatsor cananea están enterrados bajo los estratos de la ocupación israelita. El área del centro de la acrópolis está enteramente ocupado por el palacio de los reyes cananeos. El descubrimiento de este enorme complejo, que comenzó ya en anteriores campañas y del que se ha dado cuenta en estas páginas, ha continuado este año y será el objeto principal del trabajo de las excavaciones en años venideros.

En el amplio patio, del que hasta ahora se ha excavado una superficie prácticamente cuadrada de treinta metros de lado, se ha comenzado a descubrir un impresionante podio construido con piedras talladas, cuya función cúltica o civil no ha podido ser todavía determinada. Las inmediaciones de este podio monumental han sido ricas en hallazgos, como pequeños recipientes de lujosa cerámica importada de Chipre, figurillas de deidades cananeas algunas de ellas en bronce, lo mismo que una bellísima cobra en actitud de ataque.

El complejo palaciego sucumbió en una gran conflagración, cuyos vestigios fueron ya observados en anteriores campañas. El intenso calor provocó la cocción de los adobes de los muros y el resquebrajamiento de sus basamentos de basalto. Los marfiles decorados y otros ricos objetos que se encuentran entre los restos del palacio muestran el lujo de sus habitantes; en esta campaña se han seguido encontrando piezas de la excepcional caja de taracea de marfil que apareció en la III Campaña de 1992. Pero el más importante descubrimiento de esta campaña de 1995 es una gran estatua hecha en basalto, encontrada en las proximidades de la entrada al palacio. Un emblema en relieve sobre el hombro de la estatua establece su identidad como una deidad. La estatua está rota en varios fragmentos a causa del fuego que destruyó el palacio; a pesar de ello, su altura, que desde el hombro a la rodilla es de unos $120 \mathrm{cms}$., la convierte en la estatua más grande de la Edad del Bronce encontrada hasta ahora en toda la zona. Entre los múltiples fragmentos de 
otras estatuas y bellos recipientes cúlticos que se encontraron en las inmediaciones de la estatua, ha resultado una agradable sorpresa la insospechada belleza de la cabeza de faraón que ofrecemos en primicia en la figura 1 , y que acaba de ser restaurada por el equipo técnico de la Expedición Hatsor.

No menos rica en hallazgos ha resultado este año el área M, una escarpada zona siempre trabajosa de excavar y no siempre grata, que en esta ocasión ha deparado todo tipo de satisfacciones a sus excavadores. En esta área $\mathrm{M}$, situada en el centro de la ladera septentrional de la acrópolis, ya en la anterior campaña se habían descubierto indicios que permitían afirmar que la buscada conexión entre la ciudad baja y la ciudad alta en la edad del Bronce cananeo se encontraba efectivamente en ese punto. Esos vestigios consistían en una escalinata y quizás una puerta. Durante esta campaña se han descubierto otros tres escalones que descienden hacia la ciudad baja. Cerca de esa escalinata se descubrió un área pavimentada con grandes losas de basalto bellamente labradas. En el centro de esa área se alza un podio de bloques de basalto. El podio está cubierto por un enorme bloque también de basalto, cuyo peso se estima en más de dos toneladas. En el centro geométrico de ese podio hay cuatro depresiones regulares que pudieron haber soportado un trono o palio (en la figura 2 ofrecemos, también en primicia, un aspecto del podio de basalto del área M). Una línea de escaleras arranca desde el área del podio hacia el oeste. Esta escalinata y sus inmediaciones habrán de ser investigadas en campañas futuras, para lo cual se ha comenzado ya a retirar parte del talud que las cubre. El pavimento, el podio y la escalinata sucumbieron en un enorme incendio, similar en todos sus detalles al que terminó con el palacio. La identidad de los causantes de la destrucción final de la Hatsor cananea y la fecha exacta de ese desastre son dos de las principales preguntas a las que nuestras investigaciones tratarán de responder.

Como ya hemos señalado en informes de campañas anteriores, las excavaciones de Hatsor son un proyecto conjunto de la Universidad Complutense de Madrid y la Universidad Hebrea de Jerusalem (Berman Center for Biblical Archaeology) con el apoyo de la Israel Exploration Society. El proyecto tiene también la colaboración financiera de la Seltz Foundation de Nueva York y de la Fundación Rothschild. Las excavaciones tienen lugar dentro del Parque Nacional de Hatsor y cuentan con la cooperación de la Dirección de Parques 
Nacionales de Israel. Los 120 participantes en la campaña de 1995 se distribuían en los siguientes grupos: los miembros de la Misión Arqueológica Complutense a Tel Hatsor (dirigida por la que suscribe); estudiantes del Instituto de Arqueología de la Universidad Hebrea de Jerusalem; el equipo del South Eastern Baptist Seminary de North Carolina (dirigido por el Prof. Steven Andrews); un grupo de la Ambassador University de Big-Sandy, Texas (presidido por R. Silva); un equipo de voluntarios alemanes dirigido por Winnie Horst; voluntarios de varios países europeos, Canadá, América y Australia completaban el equipo de excavadores, al que se unió un contingente de trabajadores enviados por el Ministerio de Bienestar Social israelí. Las excavaciones de Hatsor están dirigidas por el Prof. Amnon Ben-Tor, de la Universidad Hebrea de Jerusalem. El equipo técnico está compuesto por A. Zarzecki (arqueóloga de campo) y los supervisores de área D. Ben-Ami, S. Cohen, J. Kolka, M. Levenshuse y Z. Zuckerman, de la Universidad Hebrea de Jerusalem y Manuel Cimadevilla, Cristina Rubio y Belén Villegas, de la Universidad Complutense de Madrid. Topografía, agrimensura y dibujo a cargo de R. Bonfil, I. Strand y M. T. Rubiato. Están a cargo de la oficina central en el campamento base T. Boaz y S. Yadid. La integración de miembros del equipo Complutense en un altamente cualificado equipo técnico es uno de los aspectos más satisfactorios de esta empresa arqueológica conjunta. 


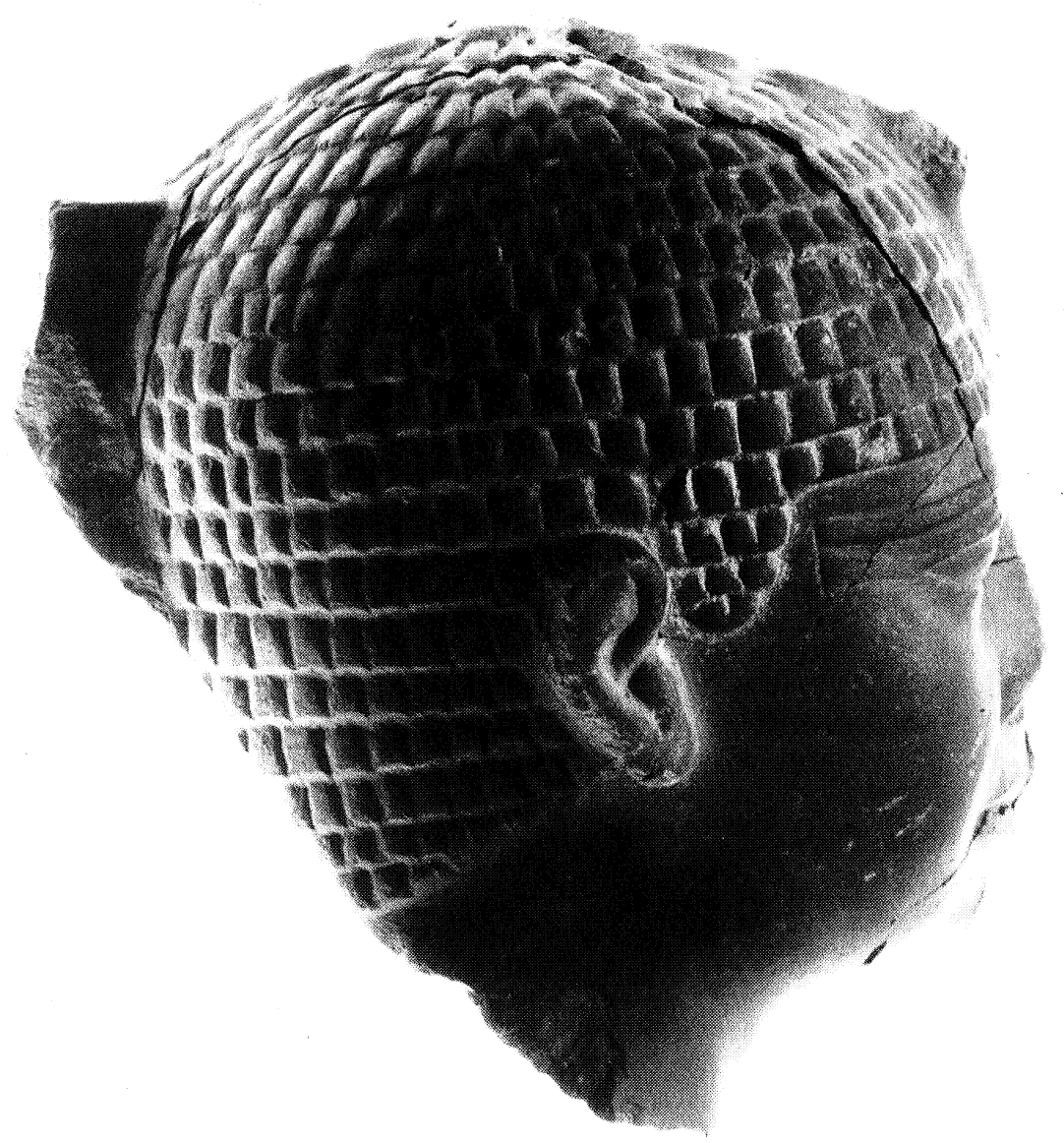

Figura 1. Cabeza de faraón 


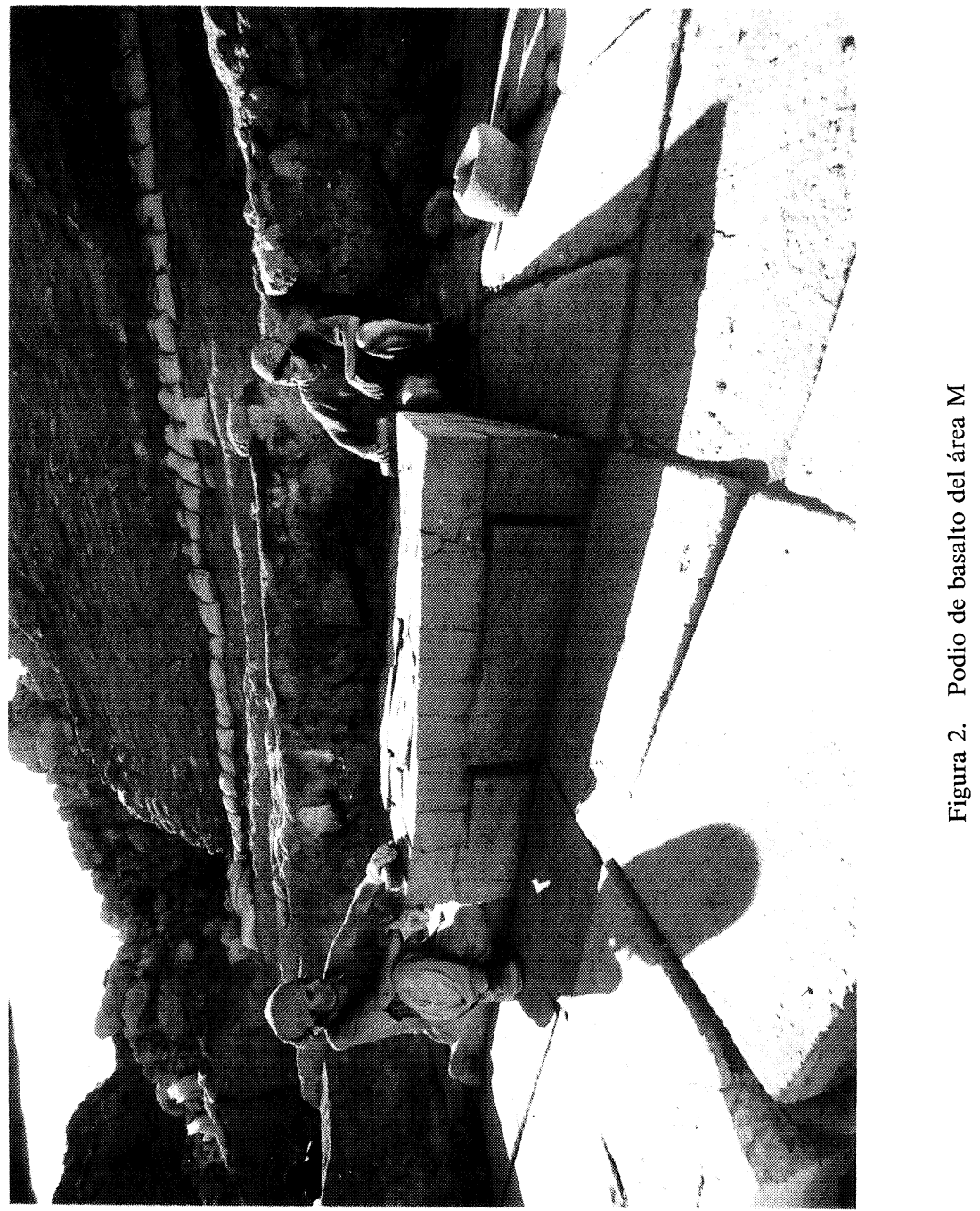




\section{RESUMEN}

La VI campaña de excavaciones arqueológicas en Tel Hatsor (1995) ha deparado importantes hallazgos que contribuyen al esclarecimiento de la historia de la ciudad en los períodos cananeo e israelita: en el plano arquitectónico, uno de los más grandes edificios públicos israelitas del siglo VIII a. C. e importantes estructuras de carácter cúltico y palaciego del Bronce Último cananeo, y entre los hallazgos materiales, el ídolo cananeo de mayor tamaño conocido y otras piezas de estatuaria cananea.

\section{SUMMARY}

The VI Archaeological Campaign in Tel Hatsor (1995) has brought important findings which contribute to enlighten the history of that city in the Cananean and Israelite periods. On the architectural grounds, it is relevant one of the biggest Israelite public buildings of the 8th century $\mathrm{BC}$ and, among other material findings, a Cananean idol - the largest known until now-, and other pieces of Cananean statuary. 